\title{
Correction: Long-term in situ permafrost thaw effects on bacterial communities and potential aerobic respiration
}

\author{
Sylvain Monteux $\mathbb{B}^{1} \cdot$ James T. Weedon $\mathbb{D}^{2,3} \cdot$ Gesche Blume-Werry ${ }^{1} \cdot$ Konstantin Gavazov ${ }^{1,4}$. \\ Vincent E. J. Jassey $\mathbb{D}^{5} \cdot$ Margareta Johansson $^{6} \cdot{\text { Frida Keuper } \mathbb{D}^{7} \cdot \text { Carolina Olid }^{1} \cdot \text { Ellen Dorrepaal }}^{1}$
}

Published online: 25 April 2019

(c) The Author(s) 2019

\section{Corrrection to: ISMEJ \\ https://doi.org/10.1038/s41396-018-0176-z \\ published online 06 June 2018}

Since the publication of the original article, the authors noticed some errors in reference citation had been introduced throughout the paper. The following text contains excerpts from the original article and how they should appear with correct referencing. The publisher apologises for any inconvenience this has caused readers.

"The limited contribution of root density to our models is in agreement with relatively small $(4 \%)$ priming effects observed in peatlands [96]."

Should read:

"The limited contribution of root density to our models is in agreement with relatively small $(4 \%)$ priming effects observed in peatlands [73]."

Sylvain Monteux

monteux@protonmail.com

1 Climate Impacts Research Centre (CIRC), Department of Ecology and Environmental Sciences, Umeå Universitet, Abisko 98107, Sweden

2 Systems Ecology, Department of Ecological Sciences, Vrije Universiteit Amsterdam, Amsterdam 1081 HV, The Netherlands

3 PLECO, Department of Biology, University of Antwerp, 2610 Wilrijk, Belgium

4 Federal Institute for Forest, Snow and Landscape Research WSL, Lausanne CH-1015, Switzerland

5 Functional Ecology and Environment Laboratory (ECOLAB), Department of Biology and Geosciences, UMR 6245 Université Toulouse III Paul Sabatier, Toulouse cedex 09 31062, France

6 Department of Physical Geography and Ecosystem Science, Lund Universitet, Lund 223 62, Sweden

7 INRA, AgroImpact UR1158, Site Laon, Barenton Bugny 02000, France
"All statistical analyses were carried out using R v3.4.1 [73], and the script used to produce figures and tables is available at https://bitbucket.org/smonteux/monteux_deep_ thaw."

Should read:

"All statistical analyses were carried out using R v3.4.1 [74], and the script used to produce figures and tables is available at https://bitbucket.org/smonteux/monteux_deep_ thaw."

"Effects of the deep-thaw treatment and soil depth on potential respiration at $11^{\circ} \mathrm{C}, \mathrm{Q} 10$, and soil abiotic variables (OMC, $\mathrm{pH}, \mathrm{NH} 4+$, and soil moisture) were assessed using analysis of variance with linear mixed-effects models (nlme package [74]), using treatment and depth as fixed factors, and core as a random factor, followed by pairwise contrasts when appropriate (1smeans package [75])."

Should read:

"Effects of the deep-thaw treatment and soil depth on potential respiration at $11^{\circ} \mathrm{C}, \mathrm{Q} 10$, and soil abiotic variables (OMC, $\mathrm{pH}, \mathrm{NH} 4+$, and soil moisture) were assessed using analysis of variance with linear mixed-effects models (nlme package [75]), using treatment and depth as fixed factors, and core as a random factor, followed by pairwise contrasts when appropriate (lsmeans package [76])."

"The effects of depth and treatment on BCS were assessed by testing for deviation of each OTU against a negative-binomial distribution with generalized linear models (manyglm(), mvabund package [76]), which confounds location and dispersion effects less than distance-based approaches (e.g., PERMANOVA [77]). A principal coordinates analysis (PCoA) ordination of weighted UniFrac distances was computed from the averaged abundance table and associations with root density and soil variables were tested using envfit() (vegan package [78])." 
Should read:

"The effects of depth and treatment on BCS were assessed by testing for deviation of each OTU against a negative-binomial distribution with generalized linear models (manyglm(), mvabund package [77]), which confounds location and dispersion effects less than distancebased approaches (e.g., PERMANOVA [78]). A principal coordinates analysis (PCoA) ordination of weighted UniFrac distances was computed from the averaged abundance table and associations with root density and soil variables were tested using envfit() (vegan package [79])."

"For both bulk and intrinsic potential respiration, we compared regression models based on their second-order Akaik's Information Criterion (AICc, AICcmodavg package [79])."

Should read:

"For both bulk and intrinsic potential respiration, we compared regression models based on their second-order Akaike's Information Criterion (AICc, AICcmodavg package [80])."

"One previous study has compared BCS at the same depth in seasonally thawed and permafrost soil in sites with permafrost degradation following fire, but there, fire and thaw effects were confounded [80]."

Should read:

"One previous study has compared BCS at the same depth in seasonally thawed and permafrost soil in sites with permafrost degradation following fire, but there, fire and thaw effects were confounded [81]."

"Eriophorum roots harbor aerenchymae, likely making deep soil more oxic (SI Fig. S4 and S7 [13, 17, 81])."

Should read:

"Eriophorum roots harbor aerenchymae, likely making deep soil more oxic (SI Fig. S4 and S7 [13, 17, 82])."

"This poorly known phylum has one cultured representative (thermophilic Caldisericum exile [82]), and has been found in other extreme environments (e.g., [83]). Wurzbacher et al. [84] recently showed that enigmatic microbial phyla dominate permafrost thaw ponds."

Should read:

"This poorly known phylum has one cultured representative (thermophilic Caldisericum exile [83]), and has been found in other extreme environments (e.g., [84]). Wurzbacher et al. [85] recently showed that enigmatic microbial phyla dominate permafrost thaw ponds."

"Upon permafrost thawing, fast metabolic responses facilitate SOM decomposition [28, 85], which could have increased carbon losses in the short term. Short periods of oxygenation, e.g., through E. vaginatum aerenchymae, can have lasting consequences on decomposition, by promoting phenol oxidase activity $[18,86]$, and seem plausible considering the observed changes in aerobic and anaerobic OTU relative abundances."

Should read:

"Upon permafrost thawing, fast metabolic responses facilitate SOM decomposition [28, 86], which could have increased carbon losses in the short term. Short periods of oxygenation, e.g., through E. vaginatum aerenchymae, can have lasting consequences on decomposition, by promoting phenol oxidase activity [18, 87], and seem plausible considering the observed changes in aerobic and anaerobic OTU relative abundances."

"This is further supported by higher Q10 values in the deep-thaw intermediate layer, suggesting increased chemical recalcitrance (SI Fig. S6 [87]). While labile C-pool depletion has been previously suggested to explain decreased soil respiration [9, 88] and would fit our observations, integrated soil respiration measurements over a long-term experiment coupled with organic matter profiling would be necessary to confirm this interpretation."

Should read:

"This is further supported by higher Q10 values in the deep-thaw intermediate layer, suggesting increased chemical recalcitrance (SI Fig. S6 [88]). While labile Cpool depletion has been previously suggested to explain decreased soil respiration [9, 89] and would fit our observations, integrated soil respiration measurements over a long-term experiment coupled with organic matter profiling would be necessary to confirm this interpretation.

"Deep thaw had contrasting effects in the permafrost layer, with decreased bulk respiration and increased intrinsic respiration. Deep thaw may raise temperatures in stillfrozen soil layers at most by $<1{ }^{\circ} \mathrm{C}$ [74]."

Should read:

"Deep thaw had contrasting effects in the permafrost layer, with decreased bulk respiration and increased intrinsic respiration. Deep thaw may raise temperatures in stillfrozen soil layers at most by $<1{ }^{\circ} \mathrm{C}$ [90]."

"We cannot rule out that microbial biomass or other soil chemistry measures, e.g., recalcitrance of SOM [89, 90], may explain additional variation in respiration."

Should read:

"We cannot rule out that microbial biomass or other soil chemistry measures, e.g., recalcitrance of SOM [91, 92], may explain additional variation in respiration." 
"However, our results are in line with recent evidence suggesting that bacterial taxonomic composition can explain a unique portion of variation in respiration (agricultural temperate soil [91]), and partly determines soil carbon dynamics [92], particularly in organic soils where microbial activity is less constrained [93, 94, 95]."

Should read:

"However, our results are in line with recent evidence suggesting that bacterial taxonomic composition can explain a unique portion of variation in respiration (agricultural temperate soil [93]), and partly determines soil carbon dynamics [94], particularly in organic soils where microbial activity is less constrained [95, 96, 97]."

"Using different experimental designs, such as root exclosures, might allow to disentangle direct and legacy effects of root colonization effects from other consequences of thawing [97]."

Should read:

"Using different experimental designs, such as root exclosures, might allow to disentangle direct and legacy effects of root colonization effects from other consequences of thawing." 